\title{
A Region Growing controlled by Edge Map technique (RGCE) for Automatic Registration of Satellite Images
}

\author{
Dr. Mohamed Ahmed Hussein
}

\begin{abstract}
Image registration is a basic and troublesome assignment where managing remotely sensed satellite images. The registration of different platform images has turned into a critical issue with an expanding number of images gathered each day from various satellites. There exist an extensive variety of registration strategies for various kinds of utilizations and information sources, however no calculation is exact for enrolling multisource images reliably. This exploration tends to this issue by examining the advancement of a completely automatic registration system for an extremely confused issue of multisensor remote detecting images particularly Synthetic aperture radar (SAR) and electrooptical images.
\end{abstract}

The development of this automatic image registration method is based on the extraction and matching of common features that are visible in both images. The algorithm involves the following five steps: noise removal, edge extraction, edge linking, pattern extraction and pattern matching to collect automatically the Ground Control Points (GCPs) required to the image registration.

The application of the developed automatic image registration model to different SAR and optical image pairs showed that accurate ground control points (GCPs) could be identified automatically.

Index Terms - SAR, Feature extraction and Matching, Automatic image registration. 
A Region Growing controlled by Edge Map technique (RGCE) for Automatic Registration of Satellite Images

\section{INTRODUCTION}

Image registration is a strategy utilizing to rectify the geometric contrast between two images at least, one of them is the called reference image and the others are the input images of a similar scene. Image registration process for the most part comprises of four primary advances. The initial step is the determination of relating highlights from both reference and input images. Case of such element are dim level forces, forms, edges, line crossing points, and factual highlights, for example, centroids [6]. Also, include coordinating is acquired between the image combine to assess the level of the match between the input image and the reference image. Procedures utilized for this reason incorporate into general: the cross relationship, the entirety of outright contrasts between the pixels, or other factual measures. Thirdly, applying the fitting change capacity to acquire the change coefficients. Such changes are unbending (turn, interpretation, and scaling); relative change, more broad than the inflexible change and tolerant of complex bending (shear and extending); and polynomial change which is utilized when questions in the input image are curved. The last advance of image registration is utilizing the change coefficients to resampling the input image to the reference image matrix to obtain the registered image.

By and large, image enrollment can be characterized as takes after: given a couple of images M1 and M2 and need to discover a closeness measure S(M1 , M2) to decide the ideal change ', with the end goal that $\mathrm{S}(\mathrm{T}(\mathrm{M} 1, \mathrm{M} 2))$ is expanded.

On the off chance that the reference image and the input image are gained from a similar sensor type, at that point the proper image registration approach is utilizing the gray level intensities of the image pair as the features and the crosscorrelation as a similarity measure. An example of this image registration approach is the registration of optical remotely sensed images of the same spectral band.

Images from different acquiring systems such as from microwaves and visible-band have different characteristics, because the image pairs come from different platforms, different sensors, different angles and different noise models. Some objects exist in one image but not in the other image and the same object appears differently in each image. This is due to the acquiring systems using different bands of the electro-magnetic spectrum. Different gray-level intensities is an image registration challenge in multi-acquisition systems in remote sensing. In dealing with this problem, point features will not be considered in registration of microwaves and visible-band images [16]. To handle image registration of microwave systems especially synthetic aperture radar (SAR) images and visibleband images, structural features should be considered. 
The issue in making a layered item from various sensors in view of various parts of electromagnetic range (say RADARSAT and Landsat) is in guaranteeing pixel to pixel enlistment, else the subsequent image's enrollment is troublesome. For this procedure to be programmed, the correlation of the two images must depend just on their substance and not require administrator choice of highlights. In this manner the basic issue is programmed include extraction and coordinating.

There is an extensive variety accordingly of various sensors to a similar surface. SAR images can be splendid where optical images are diminish and the other way around. Geography is conspicuous in RADARSAT however not Landsat, while Landsat portrays streets and waterways well yet RADARSAT does not. Hence highlight extraction must be extremely segregating to look at images caught by various sensors. Rignot et al. [4] have demonstrated that some order plans can fragment images from various sensors into tantamount locales. So also, edge identification calculations that are harsh to the SAR dot commotion in SAR, will deliver direct highlights that think about well between images.

Following determination of regular highlights, their areas must be geometrically coordinated to decide the ideal enlistment revision. This should be possible in various ways. The highlights can be spoken to as parallel images and territory relationship connected to discover the misregistration shifts. Different techniques, known as separation change and chamfer coordinating [3] (bend coordinating utilizing separation capacities), utilize a summed up separation to gauge the confound between highlights. Improving this separation as a component of move decides the rectification. These strategies function admirably if the highlights are shut fit as a fiddle and introduction. For all the more extremely bungled highlights, dynamic programming [7] and autoregressive model techniques might be attempted. In any case, the matches are found between examples of the diverse images through certain obliges [4][5]. Procedures for robotized multi-sensor image enlistment are still in their early stages. Strategies are at exhibit specially appointed and specifically connected as required by the information under thought.

In spite of the fact that the images to be enrolled are procured from a similar scene, there are two essential contrasts between them: the qualities of the sensors (extending and sheering) and a conceivable observable interpretation, pivot and scaling of the scenes because of the diverse places of these sensors. Anyway SAR and optical images of a similar scene show up very unique as found in Fig. 1. In this manner the initial phase in endeavoring to enlist them is to extricate only the 
basic highlights in them two. In this paper, the improvement of this new programmed image enrollment technique depends on the extraction and coordinating of basic highlights that are unmistakable in the two Images.
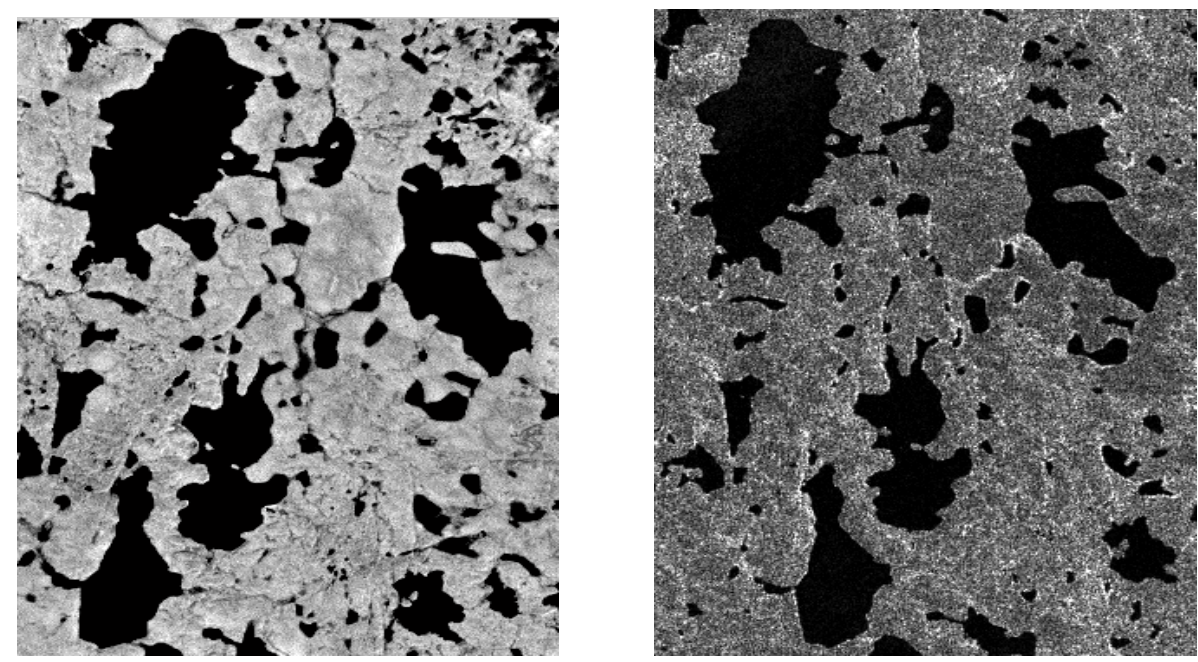

Fig. 1 Original Optical image (Landsat) and original SAR image

The main goal of this paper is to design and develop fast and accurate techniques for the automatic registration of microwave-based (synthetic aperture radar, (SAR) and interferometric SAR), and visible-band remotely sensed images. This is achieved by the automatic selection of the corresponding control points. To achieve this goal some other objectives were considered.

- Investigate noise removing filters to enhance the feature extraction process for the given data.

- Edge extraction algorithms is considered to obtain accurate edge map for the given data.

- Object extraction techniques is essential to develop an accurate registration to be used with different data.

A background on two-dimensional image registration is in Section 2, The developed algorithm involves the following five steps: noise removal edge extraction, edge linking, the pattern extraction using controlled region growing and pattern matching in Section 3. A discussion and some concluding remarks are given in Section 4. 


\section{Two-Dimensional Image Registration}

Algorithms for image registration can be classified into two categories: feature- based and area-based algorithms. The feature-based approach extracts common features such as areas, curves, lines or patches from each image and uses them to perform an accurate registration. Such an approach has proved to be more suitable for problems of multi-sensor image registration [6] [11] [12] [13] [14] [15] since most of those features do not depend on the gray-level characteristics.

The area-based registration approach uses a statistical measures to judge if one area in the input image has a correspondence in the reference image and vice-versa. The image pair is divided into small equal areas which are compared pairwise, depending on the gray level intensities of these areas [6]. The areas which have similarity are considered to be matched and from these matched areas control points can be extracted.

The determination of the corresponding control points in the images is a difficult process. At the same time it is the main step that can determine the accuracy of the registration process. Therefore, most researchers have focused on determining methods to loca.te control points in the images with the highest possible accuracy, as well as finding the correspondence between these control points [6]. The automation of image registration will be discussed in the next two sections.

\subsection{Manual Image Registration}

Manual image registration is characterized by the manual selection of the corresponding control points. In the field of remote sensing these control points are called ground control points (GCPs). The processing chain of such a registration is summarized by the following four steps: visually selecting the corresponding GCPs from each image, selecting the transformation function, determining tie parameters of the transformation function by using the GCPs, and using the different resampling approaches to resample the input image to the reference image by employing the transformation coefficients.

\subsection{Automatic Image Registration}

Very few automatic image registration algorithms have been developed by researchers in remote sensing. An optimal solution to multi-modal automatic image registration has not been designed. This is due to the difficulties in determining the best way for generating accurate GCPs automatically.

To perform image registration automatically, it is necessary to divide the first step of manual image registration into two steps during the registration process: feature extraction and feature matching. 
A Region Growing controlled by Edge Map technique (RGCE) for Automatic Registration of Satellite Images

\section{RGCE automatic registration Technique}

The proposed Region Growing Controlled by Edge Map (RGCE) technique is consists of five steps. The first step is noise removal followed by edge extraction and to overcome the problem of uncompleted edges the edge linking is performed. Pattern extraction and pattern matching are performed to collect the Ground Control points (GCPs) automatically as discussed in the following. Fig. 2 illustrates the RGCE Model.

\subsection{SAR preprocessing}

SAR image products are corrupted by speckle noise, which compromises the radiometric quality of the image and hence reduces the signal to noise ratio. In SAR imagery, speckle is generally modeled as a multiplicative noise. SAR images are useful, but the corrupting speckle makes the interpretation difficult. To improve the interpretability, and therefore the usefulness of SAR images, it is essential to try to remove or reduce the speckle. A very large number of speckle reduction filters have been developed over the years [8]. Some of the groups of speckle reduction filters are simple filters, rigorous adaptive filters, non-rigorous adaptive filters and modified traditional filters. However, selecting the best SAR filter depends on features of

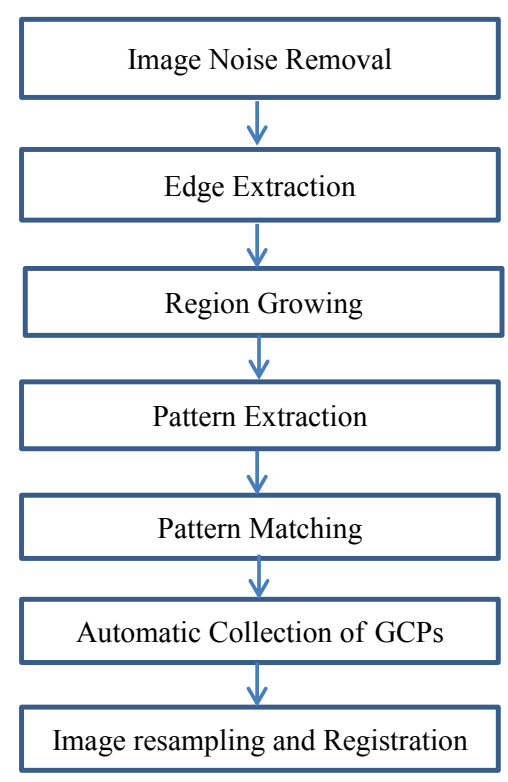

Fig. 2 RGCE Model Block Diagram 
Journal of the ACS, Vol. 10, May 2019

interest. In this application, features must have closed boundaries and they must not be too small, since small features are difficult to match. Therefore the ideal speckle reduction filters for this application can remove or reduce the speckle while retain features with strong edges. The standard speckle filters such as Statistical Lee, Kuan, Gemma or MAP, Standard Frost, and Entranced Frost are primarily used on radar data to remove speckle effect. The Kuan filter was selected for this task [1].

\subsection{Optical preprocessing}

In contrast to SAR data, optical data requires very little radiometric preprocessing before feature extraction. Since the signal to noise ratio is typically high, noise does not obscure features and generally no smoothing is required. However, application of histogram equalization making features become much more distinct from their backgrounds and improved the success of optical image feature extraction algorithms.

\subsection{EDGE EXTRACTION}

Most remote sensing applications, such as image registration, image segmentation, region separation, object description and recognition use edge detection as a preprocessing stage for feature extraction. Image edges are usually found where there is a sudden change in image intensity. This will result in local minima or maxima of the first derivative of the intensity. Equivalently, this same location will have a zero-crossing of the second derivative.

The edge detector should provide consistent identification of high contrast boundaries. A number of different edge extraction schemes have been considered [9]. A modified Canny edge detector was considered suitable [2][10], and this filter is demonstrated in Fig. 3.

\subsection{EDGE LINKING}

Since the contours within the edge map are often incomplete, some forms of edge linking is necessary. Morphological operators are applied to obtain closed contours. The edge map is dilated using a window proportional to the gap size to achieve the closed contours. This operation is necessary for accurate pattern extraction. 
A Region Growing controlled by Edge Map technique (RGCE) for Automatic Registration of Satellite Images
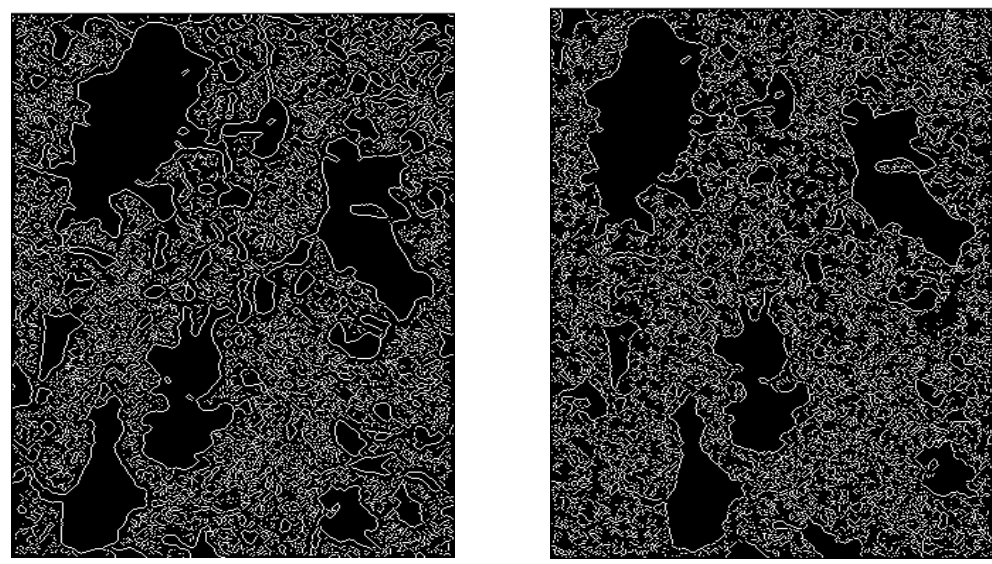

Fig. 3 Edge maps using a modified Canny edge detector

\subsection{PATTERN EXTRACTION}

Automatic feature based mostly image registration of various sorts of images involves 2 main challenges: feature extraction and have matching. The success of the matching method depends primarily on the accuracy of the feature extraction method. Therefore, researchers still target developing feature extraction algorithms that change the extraction of appropriate options from every image to be matched.

Any two-dimensional patch in an image is simply called an object. It is much more difficult for a computer to perform the object extraction. To extract the object features from a given image, a segmentation technique should be applied.

Pattern extraction is performed to identify large homogeneous regions within closed edge map. The initial regions are obtained by scanning the edge map with a square window and masking pixels when the window is completely free of edge points. These initial regions are then grown until they meet the enclosing edge contours. This process identifies the large edge free regions while ignoring smaller, less significant features.

\subsection{PATTERN MATCHING}

After region growing is completed, the patterns are assigned arbitrary labels. The final step of the overall algorithm, pattern matching, is performed. This is done by using the attributes of the patterns in both images such as perimeter, centroid, area, convex hull, number of pixels above horizontal center line, number of pixels to the right of vertical center line and the area of bounding box of the pattern. The difference between the attributes determines the value of a matching cost function $C$. The matching function is determined for the first pattern in reference image and all the patterns in input image to be registered. The combination of patterns 
Journal of the ACS, Vol. 10, May 2019

which has minimum matching cost is accepted as the best match. Pattern $A$ from the first image and $B$ from the second image are selected as a matched pair if the following conditions are satisfied:

a) $C_{A B} \leq C_{A B^{\prime}}$ ie. $B^{\prime}$ Includes all the patterns with similar shapes to pattern $A$,

(b) $C_{A B}<T$ ie. If the minimum matching cost is above the threshold, $T$, there is no match.
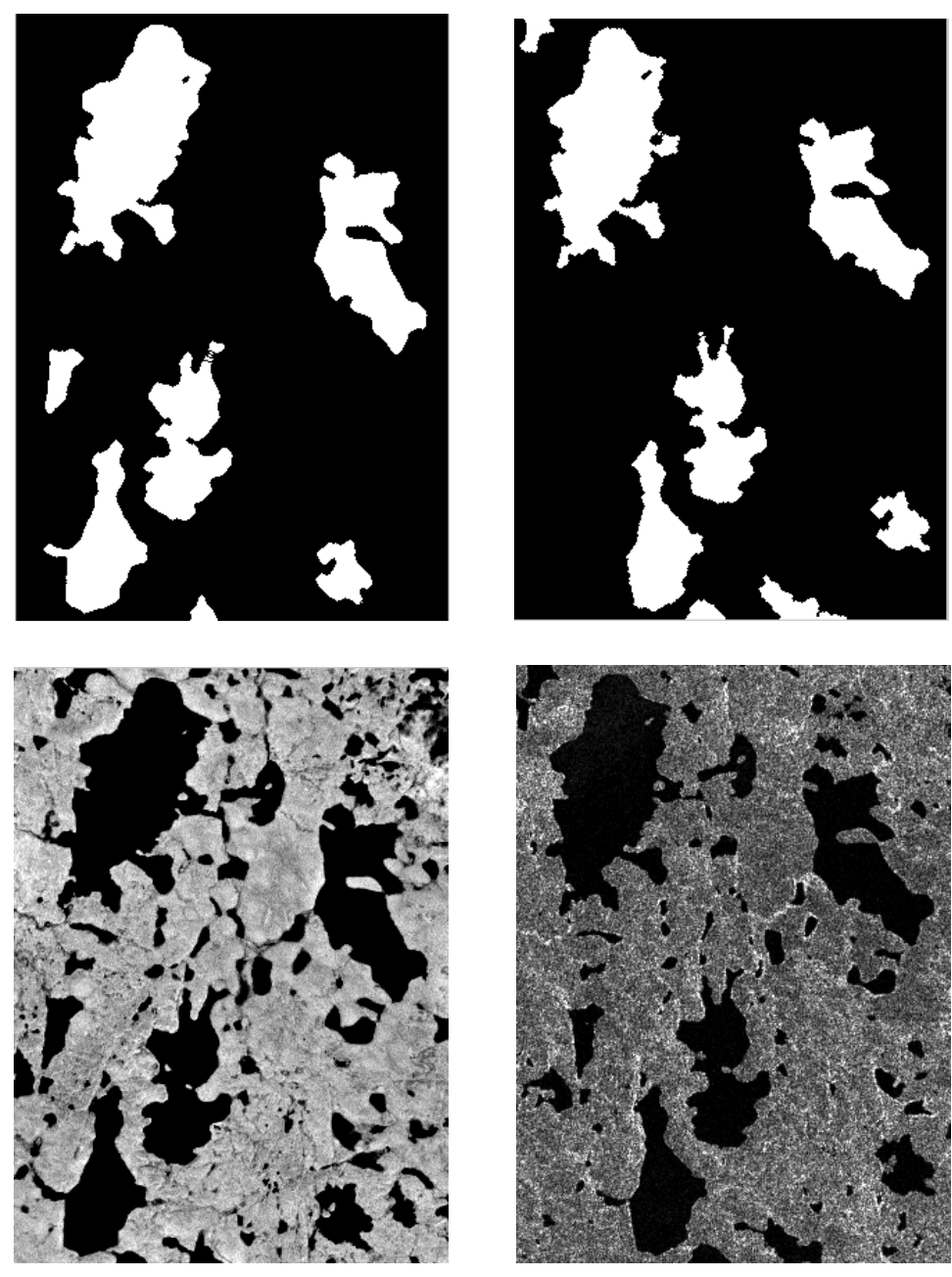

Fig. 4 Extracted patterns from Landsat (a) and SAR (b) images. (c) and (d) are the original Landsat and SAR 
The process is "pairwise exhaustive" ie. repeating for the second, third and all subsequent patterns in reference image until they have all been matched with patterns in input image. In the situation where a pattern from input image has been matched with two different patterns from reference image, the match with the lowest match function is accepted as the correct one. The result is that all the patterns in reference image have been matched, but not necessarily all of the patterns in input image. To ensure that all of the patterns in input image have the opportunity to be matched with all of the patterns in reference image, the process is repeated with the order of the images reversed. The first pattern in input image is matched with all the patterns in reference image, as is the second, third, and so on. Multiple matches are again eliminated using the value of the matching function. The centroid of the matched patterns are taken as accurate ground control points (GCPs). The application of the developed automatic image registration model to a SAR (ERS-1), SPOT pair images and RADARSAT and Landsat pair images showed that (GCPs) can be identified automatically, as displayed in Fig. 4, Fig 5 and Fig 6. Following the GCP recognition, standard methods can be used for registration.

To assess the exactness of the registration comes about utilizing the RGCE model, all the substantial GCPs are taken into assessment. Root Mean Square Error (RMSE) including all the GCPs is 0.2579 pixels in the $\mathrm{X}$ direction and 0.2327 pixels in the $\mathrm{Y}$ direction.

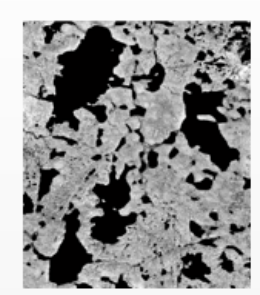

Original Landsat image

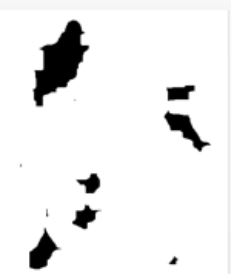

Start patches extraction

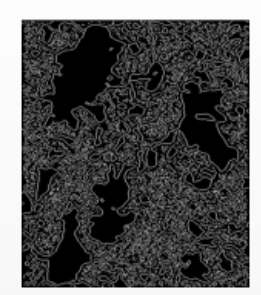

Edge map

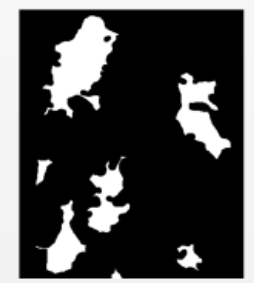

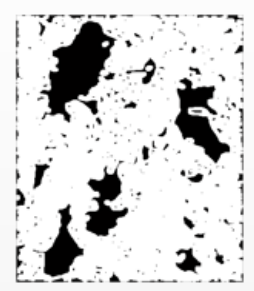

After dilation

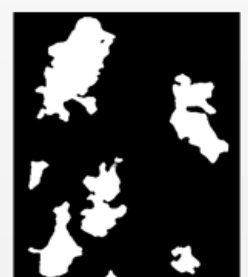

Extracted patches

Fig. 5 Application the RGCE algorithm on Landsat image 


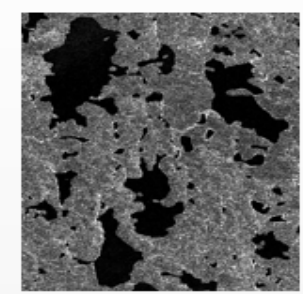

Original SAR image
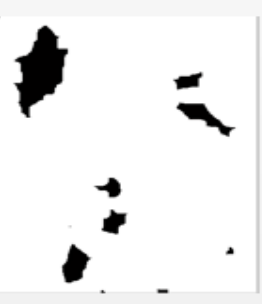

Start patches extraction

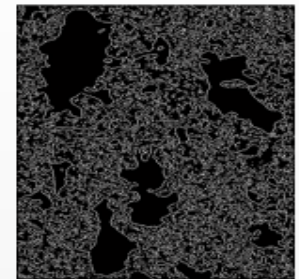

Edge map

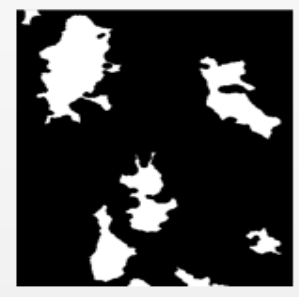

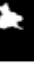

Fig. 6 Application the RGCE algorithm on SAR image

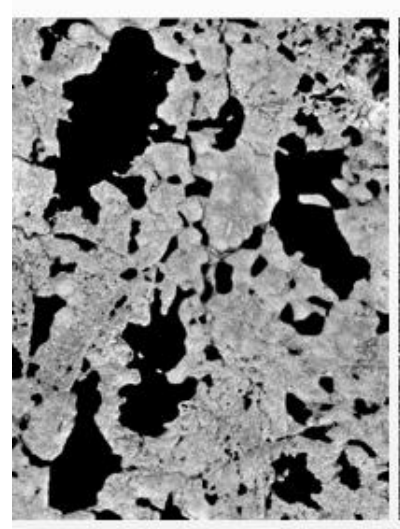

Landsat Image

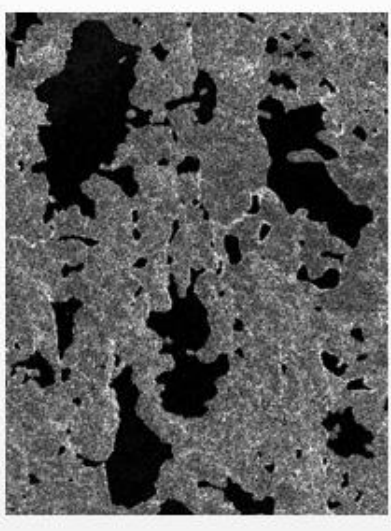

RADARSAT Image

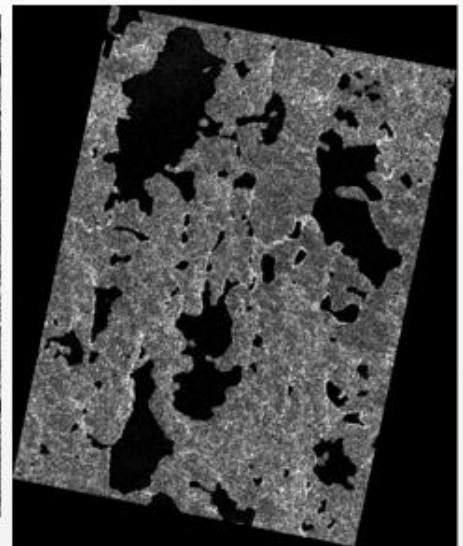

Registered SAR

Fig. 7 Landsat and Radarsat SAR images and registered SAR image 


\section{CONCLUSION}

The proposed region growing controlled by the edge map (RGCE) object extraction method is a very efficient method for object extraction from both SAR and visible-band images. One important thing for measuring the efficiency of the RGCE algorithm with respect to time is the selection of tie seeds for the region growing. The size of the seed is big (produced from the scanning mask) which speeds up the growing process. To evaluate tie RGCE algorithm it is applied on different remotely sensed images and the objects extracted by this technique are much more accurate in shape and dimension than thresholding, homogenous regions, and segmentation techniques. There are no limitations on shape or dimension of objects to be extracted, but for accuracy very small objects are not considered by setting on appropriate threshold value.

The region growing controlled by edge outline more exact than utilizing image segmentation for pattern extraction because of the vulnerability of division limits. Implementation of the proposed model has demonstrated that automatic ground control points measurement between sets of SAR and optical images can be precisely accomplished as illustrated in Fig. 7.

\section{REFERENCES}

[1] D. T. Kuan, A. A. Sawchuk, T. C. Strand, P. Chavel, "Adaptive noise smoothing filter for images with signal-dependent noise", IEEE Trans. Patt. Anal. Machine Intel, Vol. 7, No. 2, pp. 165-177, 1985.

[2] J. F. Canny,"A computational approach to edge detection," IEEE Trans. Pattern Analysis and Machine Intelligence, Vol: 8, pp. 679-698, 1986.

[3] B Gunilla, "Hierarchical Chamfer Matching: A Parametric Edge Matching Algorithm", IEEE Transactions on Pattern Analysis and Machine Intelligence, Vol. 10, No. 6, pp. 849 - 865, Nov. 1988.

[4] E. J. M, Rignot, R. Kwok, J. C. Curlander and S. S. Pang," Automated multisensor registration: Requirements and techniques", Photogrammetric Engineering and Remote Sensing, Vol. 57, pp. 1029-1038, 1991.

[5] J. C. Curlander, and R. N. McDonough, "Synthetic Aperture Radar, Systems and Signal Processing," John Wiley \& Sons, New York, 1991. 
[6] L. G Brown. "A Survey of Image Registration Techniques". ACM Computer Surveys, 24(4):325-376,1992.

[7] G. M. Qu'enot, "Image Matching using Dynamic Programming: Application to Stereovision and Image Interpolation" Image Communication, Bordeaux, France, 20-22 may 1996.

[8] I. McConnell and C. Oliver, "Comparison of annealing and iterated filters for speckle reduction in SAR", in Microwave Sensing and Synthetic Aperture Radar, Proc. SPIE 2958 ,pp.74-85,1996.

[9] M. Heath, S. Sarkar, T. Sanocki, and K. Bowyer, "Comparison of Edge Detectors: A Methodology and Initial Study," Proc. IEEE Conf. Computer Vision Pattern Recognition, San Francisco, IEEE CS Press, pp. 143-148, June 1996.

[10] M. Ali, and D. Clausi "Using the Canny edge detector for feature extraction and enhancement of remote sensing images", Proceedings of International Geoscience and Remote Sensing Symposium, IGARSS, Sydney, Australia, 913 July 2001.

[11] Suma Dawn, Vikas Saxena, Bhudev Sharma “ Remote Sensing Image Registration techniques: A Survey", International Conference on Image and Signal Processing ICISP 2010: Image and Signal Processing pp 103-112.

[12] Dr. H.B.Kekre, Dr. Tanuja K. Sarode and Ms. Ruhina B. Karani. "2D Satellite Image Registration Using Transform Based and Correlation Based Methods". International Journal of Advanced Computer Science and Applications, Vol. 3, No. 5, 2011.

[13] Heena R. Kher. "Implementation of Image Registration for Satellite Images using Mutual Information and Particle Swarm Optimization Techniques". International Journal of Computer Applications (0975 - 8887) Volume 97No.1, July 2014.

[14] Ms. Parita P. Vaghasiya, Pankaj Kumar Gautam.” Image Registration Techniques: A Review". International Journal Of Engineering And Computer Science ISSN:2319-7242 Volume 4 Issue 2 February 2015, Page No. 10489-10492.

[15] C. P. Dalmiya1* and V. S. Dharun. "A Survey of Registration Techniques in Remote Sensing Images". Indian Journal of Science and Technology, Vol 8(26), DOI: $10.17485 /$ ijst/2015/v8i26/81048, October 2015.

[16] Haigang Sui, Zhina Song, Dongsheng Gao, Dongsheng Gao. "Automatic Image Registration Based on Shape Features and Multi-scale Image Segmentation" Multimedia and Image Processing (ICMIP), 2017 2nd International Conference 